CERN PPE/94-93

15 June 1994

\title{
Observation of monojet events and tentative interpretation
}

\author{
The ALEPH Collaboration*)
}

\begin{abstract}
A data sample corresponding to almost two million hadronic $\mathrm{Z}$ decays collected by the ALEPH detector at LEP has been searched for monojet events. Three events were found, in agreement with the expectation from the process $\mathrm{e}^{+} \mathrm{e}^{-} \rightarrow \gamma^{*} \nu \bar{\nu}$, with $\gamma^{*} \rightarrow \mathrm{ff}$. Two events are hadronic, the third one being an $\mathrm{e}^{+} \mathrm{e}^{-}$pair. All monojet masses are in excess of $3 \mathrm{GeV} / c^{2}$, and two of the events have large transverse momenta: 18.5 and $20.3 \mathrm{GeV} / c$. These kinematic characteristics are quite unlikely in the process $\mathrm{e}^{+} \mathrm{e}^{-} \rightarrow \gamma^{*} \nu \bar{\nu}$. The probability of their occurrence increases substantially when processes involving further $\mathrm{Z}$ or W exchanges are taken into account, but still remains at the $5 \%$ level.
\end{abstract}

(Submitted to Physics Letters B)

*) See next pages for the list of authors. 


\section{The ALEPH Collaboration}

D. Buskulic, D. Casper, I. De Bonis, D. Decamp, P. Ghez, C. Goy, J.-P. Lees, M.-N. Minard, P. Odier,

B. Pietrzyk

Laboratoire de Physique des Particules (LAPP), IN P $^{3}$-CNRS, 74019 Annecy-le-Vieux Cedex, France

F. Ariztizabal, M. Chmeissani, J.M. Crespo, I. Efthymiopoulos, E. Fernandez, M. Fernandez-Bosman, V. Gaitan, Ll. Garrido, ${ }^{28}$ M. Martinez, T. Mattison, ${ }^{29}$ S. Orteu, A. Pacheco, C. Padilla, F. Palla, A. Pascual, J.A. Perlas, F. Teubert

Institut de Fisica d'Altes Energies, Universitat Autonoma de Barcelona, 08193 Bellaterra (Barcelona), $\operatorname{Spain}^{7}$

D. Creanza, M. de Palma, A. Farilla, G. Iaselli, G. Maggi, N. Marinelli, S. Natali, S. Nuzzo, A. Ranieri,

G. Raso, F. Romano, F. Ruggieri, G. Selvaggi, L. Silvestris, P. Tempesta, G. Zito

Dipartimento di Fisica, INFN Sezione di Bari, 70126 Bari, Italy

Y. Chai, D. Huang, X. Huang, J. Lin, T. Wang, Y. Xie, D. Xu, R. Xu, J. Zhang, L. Zhang, W. Zhao Institute of High-Energy Physics, Academia Sinica, Beijing, The People's Republic of China ${ }^{8}$

G. Bonvicini, J. Boudreau, ${ }^{25}$ P. Comas, P. Coyle, H. Drevermann, A. Engelhardt, R.W. Forty, G. Ganis, C. Gay, ${ }^{3}$ M. Girone, R. Hagelberg, J. Harvey, R. Jacobsen, B. Jost, J. Knobloch, I. Lehraus, M. Maggi, C. Markou, E.B. Martin, P. Mato, H. Meinhard, A. Minten, R. Miquel, P. Palazzi, J.R. Pater, P. Perrodo, J.-F. Pusztaszeri, F. Ranjard, L. Rolandi, J. Rothberg, ${ }^{2}$ M. Saich, ${ }^{6}$ D. Schlatter, M. Schmelling, W. Tejessy, I.R. Tomalin, R. Veenhof, A. Venturi, H. Wachsmuth, S. Wasserbaech, ${ }^{2}$ W. Wiedenmann, T. Wildish, W. Witzeling, J. Wotschack

European Laboratory for Particle Physics (CERN), 1211 Geneva 23, Switzerland

Z. Ajaltouni, M. Bardadin-Otwinowska, A. Barres, C. Boyer, A. Falvard, P. Gay, C. Guicheney, P. Henrard, J. Jousset, B. Michel, J-C. Montret, D. Pallin, P. Perret, F. Podlyski, J. Proriol, F. Saadi Laboratoire de Physique Corpusculaire, Université Blaise Pascal, IN ${ }^{2} P^{3}$ CNRS, Clermont-Ferrand, 63177 Aubière, France

T. Fearnley, J.B. Hansen, J.D. Hansen, J.R. Hansen, P.H. Hansen, S.D. Johnson, R. Møllerud, B.S. Nilsson

Niels Bohr Institute, 2100 Copenhagen, Denmark ${ }^{9}$

A. Kyriakis, E. Simopoulou, I. Siotis, A. Vayaki, K. Zachariadou

Nuclear Research Center Demokritos (NRCD), Athens, Greece

A. Blondel, G. Bonneaud, J.C. Brient, P. Bourdon, L. Passalacqua, A. Rougé, M. Rumpf, R. Tanaka,

A. Valassi, M. Verderi, H. Videau

Laboratoire de Physique Nucléaire et des Hautes Energies, Ecole Polytechnique, IN ${ }^{2} P^{3}$-CNRS, 91128

Palaiseau Cedex, France

D.J. Candlin, M.I. Parsons, E. Veitch

Department of Physics, University of Edinburgh, Edinburgh EH9 3JZ, United Kingdom ${ }^{10}$

E. Focardi, G. Parrini

Dipartimento di Fisica, Università di Firenze, INFN Sezione di Firenze, 50125 Firenze, Italy

M. Corden, M. Delfino, ${ }^{12}$ C. Georgiopoulos, D.E. Jaffe, D. Levinthal ${ }^{15}$

Supercomputer Computations Research Institute, Florida State University, Tallahassee, FL 323064052, USA ${ }^{13,14}$

A. Antonelli, G. Bencivenni, G. Bologna, ${ }^{4}$ F. Bossi, P. Campana, G. Capon, F. Cerutti, V. Chiarella, G. Felici, P. Laurelli, G. Mannocchi, ${ }^{5}$ F. Murtas, G.P. Murtas, M. Pepe-Altarelli, S. Salomone

Laboratori Nazionali dell'INFN (LNF-INFN), 00044 Frascati, Italy 
P. Colrain, I. ten Have, I.G. Knowles, J.G. Lynch, W. Maitland, W.T. Morton, C. Raine, P. Reeves, J.M. Scarr, K. Smith, M.G. Smith, A.S. Thompson, S. Thorn, R.M. Turnbull

Department of Physics and Astronomy, University of Glasgow, Glasgow G12 8QQ, United Kingdom ${ }^{10}$

U. Becker, O. Braun, C. Geweniger, P. Hanke, V. Hepp, E.E. Kluge, A. Putzer, ${ }^{1}$ B. Rensch, M. Schmidt, H. Stenzel, K. Tittel, M. Wunsch

Institut für Hochenergiephysik, Universität Heidelberg, 69120 Heidelberg, Fed. Rep. of Germany ${ }^{16}$

R. Beuselinck, D.M. Binnie, W. Cameron, M. Cattaneo, D.J. Colling, P.J. Dornan, J.F. Hassard, N. Konstantinidis, L. Moneta, A. Moutoussi, J. Nash, D.G. Payne, G. San Martin, J.K. Sedgbeer, A.G. Wright

Department of Physics, Imperial College, London SW7 2BZ, United Kingdom ${ }^{10}$

P. Girtler, D. Kuhn, G. Rudolph, R. Vogl

Institut für Experimentalphysik, Universität Innsbruck, 6020 Innsbruck, Austria ${ }^{18}$

C.K. Bowdery, T.J. Brodbeck, A.J. Finch, F. Foster, G. Hughes, D. Jackson, N.R. Keemer, M. Nuttall, A. Patel, T. Sloan, S.W. Snow, E.P. Whelan

Department of Physics, University of Lancaster, Lancaster LA1 4YB, United Kingdom ${ }^{10}$

A. Galla, A.M. Greene, K. Kleinknecht, J. Raab, B. Renk, H.-G. Sander, H. Schmidt, S.M. Walther, R. Wanke, B. Wolf

Institut für Physik, Universität Mainz, 55099 Mainz, Fed. Rep. of Germany ${ }^{16}$

A.M. Bencheikh, C. Benchouk, A. Bonissent, D. Calvet, J. Carr, C. Diaconu, F. Etienne, D. Nicod, P. Payre, L. Roos, D. Rousseau, P. Schwemling, M. Talby

Centre de Physique des Particules, Faculté des Sciences de Luminy, IN ${ }^{2} P^{3}$-CNRS, 13288 Marseille, France

S. Adlung, R. Assmann, C. Bauer, W. Blum, D. Brown, P. Cattaneo, ${ }^{23}$ B. Dehning, H. Dietl, F. Dydak, ${ }^{21}$ M. Frank, A.W. Halley, K. Jakobs, H. Kroha, J. Lauber, G. Lütjens, G. Lutz, W. Männer, H.-G. Moser, R. Richter, J. Schröder, A.S. Schwarz, R. Settles, H. Seywerd, U. Stierlin, ${ }^{30}$ U. Stiegler, R. St. Denis, G. Wolf

Max-Planck-Institut für Physik, Werner-Heisenberg-Institut, 80805 München, Fed. Rep. of Germany ${ }^{16}$

R. Alemany, J. Boucrot, O. Callot, A. Cordier, F. Courault, M. Davier, L. Duflot, J.-F. Grivaz, Ph. Heusse, M. Jacquet, P. Janot, D.W. Kim ${ }^{19}$ F. Le Diberder, J. Lefrançois, A.-M. Lutz, G. Musolino, I. Nikolic, H.J. Park, I.C. Park, M.-H. Schune, S. Simion, J.-J. Veillet, I. Videau

Laboratoire de l'Accélérateur Linéaire, Université de Paris-Sud, IN ${ }^{2} P^{3}$ CNRS, 91405 Orsay Cedex, France

D. Abbaneo, G. Bagliesi, G. Batignani, U. Bottigli, C. Bozzi, G. Calderini, M. Carpinelli, M.A. Ciocci, V. Ciulli, R. Dell'Orso, I. Ferrante, F. Fidecaro, L. Foà, ${ }^{1}$ F. Forti, A. Giassi, M.A. Giorgi, A. Gregorio, F. Ligabue, A. Lusiani, P.S. Marrocchesi, A. Messineo, G. Rizzo, G. Sanguinetti, P. Spagnolo, J. Steinberger, R. Tenchini, ${ }^{1}$ G. Tonelli, ${ }^{27}$ G. Triggiani, C. Vannini, P.G. Verdini, J. Walsh

Dipartimento di Fisica dell'Università, INFN Sezione di Pisa, e Scuola Normale Superiore, 56010 Pisa, Italy

A.P. Betteridge, Y. Gao, M.G. Green, D.L. Johnson, P.V. March, T. Medcalf, Ll.M. Mir, I.S. Quazi, J.A. Strong

Department of Physics, Royal Holloway \& Bedford New College, University of London, Surrey TW20 OEX, United Kingdom ${ }^{10}$

V. Bertin, D.R. Botterill, R.W. Clifft, T.R. Edgecock, S. Haywood, M. Edwards, P.R. Norton, J.C. Thompson

Particle Physics Dept., Rutherford Appleton Laboratory, Chilton, Didcot, Oxon OX11 OQX, United Kingdom ${ }^{10}$ 
B. Bloch-Devaux, P. Colas, H. Duarte, S. Emery, W. Kozanecki, E. Lançon, M.C. Lemaire, E. Locci, B. Marx, P. Perez, J. Rander, J.-F. Renardy, A. Rosowsky, A. Roussarie, J.-P. Schuller, J. Schwindling, D. Si Mohand, B. Vallage

CEA, DAPNIA/Service de Physique des Particules, CE-Saclay, 91191 Gif-sur-Yvette Cedex, France ${ }^{17}$

R.P. Johnson, A.M. Litke, G. Taylor, J. Wear

Institute for Particle Physics, University of California at Santa Cruz, Santa Cruz, CA 95064, USA ${ }^{22}$

A. Beddall, C.N. Booth, S. Cartwright, F. Combley, I. Dawson, A. Koksal, C. Rankin, L.F. Thompson Department of Physics, University of Sheffield, Sheffield S3 7RH, United Kingdom ${ }^{10}$

A. Böhrer, S. Brandt, G. Cowan, ${ }^{1}$ E. Feigl, C. Grupen, G. Lutters, J. Minguet-Rodriguez, F. Rivera, ${ }^{26}$ P. Saraiva, U. Schäfer, L. Smolik

Fachbereich Physik, Universität Siegen, 57068 Siegen, Fed. Rep. of Germany ${ }^{16}$

L. Bosisio, R. Della Marina, G. Giannini, B. Gobbo, L. Pitis, F. Ragusa ${ }^{20}$

Dipartimento di Fisica, Università di Trieste e INFN Sezione di Trieste, 34127 Trieste, Italy

L. Bellantoni, J.S. Conway, ${ }^{24}$ Z. Feng, D.P.S. Ferguson, Y.S. Gao, J. Grahl, J.L. Harton, O.J. Hayes, H. Hu, J.M. Nachtman, Y.B. Pan, Y. Saadi, M. Schmitt, I. Scott, V. Sharma, J.D. Turk, A.M. Walsh, F.V. Weber, ${ }^{1}$ Sau Lan Wu, X. Wu, J.M. Yamartino, M. Zheng, G. Zobernig

Department of Physics, University of Wisconsin, Madison, WI 53706, USA ${ }^{11}$

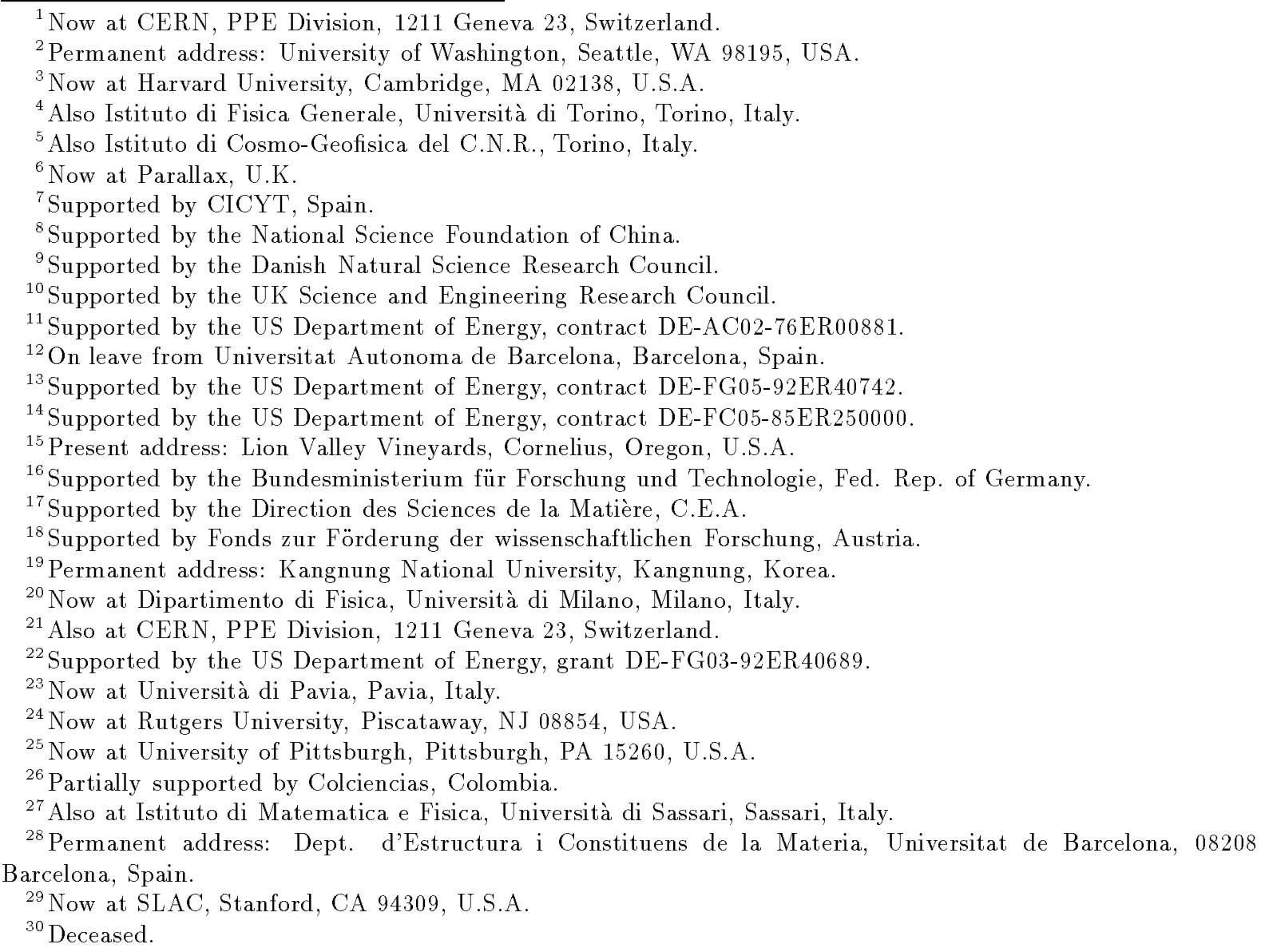


The monojet topology is commonly accepted as being background free for new particle searches in $\mathrm{e}^{+} \mathrm{e}^{-}$collisions. It has been considered, for instance, as providing a clear signature for the production of a light Higgs boson in the reaction $\mathrm{e}^{+} \mathrm{e}^{-} \rightarrow \mathrm{H} \nu \bar{\nu}$, or of light neutralinos in the reaction $\mathrm{e}^{+} \mathrm{e}^{-} \rightarrow \chi \chi^{\prime}$ (with $\chi^{\prime} \rightarrow \chi \mathrm{Z}^{*}$ ). Many unsuccessful searches[1] have been made until last year when, in the context of a search for an invisible Higgs boson, a clean monojet-like $\mathrm{e}^{+} \mathrm{e}^{-}$pair was reported by the ALEPH Collaboration[2], with a mass of $3.3 \mathrm{GeV} / c^{2}$ and a transverse momentum $p_{T}$ of $20.3 \mathrm{GeV} / c$ with respect to the beam axis. An interpretation for this event as arising from the reaction $\mathrm{e}^{+} \mathrm{e}^{-} \rightarrow \gamma^{*} \nu \bar{\nu}$, with $\gamma^{*} \rightarrow \mathrm{e}^{+} \mathrm{e}^{-}$, was proposed, but the probability that this process lead to such high mass and high $p_{T}$ values was estimated to be at the $2 \%$ level.

In this letter, the search for monojets has been extended to the full data sample collected by ALEPH from 1989 to 1993, corresponding to 1.94 million hadronic Z decays and to an integrated luminosity of $82 \mathrm{pb}^{-1}$, at energies at and close to the $\mathrm{Z}$ peak. A detailed description of the ALEPH detector can be found in Ref. 3. The features particularly relevant for this analysis are:

- a tracking system consisting of a two-layer silicon vertex detector (VDET), an eightlayer cylindrical drift chamber (ITC) and a large time projection chamber (TPC) providing up to 21 space coordinates;

- hermetic energy detection provided by a fine grained electromagnetic calorimeter (ECAL), located inside the superconducting coil, by a hadronic calorimeter (HCAL), and by luminosity calorimeters down to polar angles with respect to the beam axis of $40 \mathrm{mrad}$ in 1989-1992 and of $24 \mathrm{mrad}$ since 1993.

Typical detection thresholds are smaller than $100 \mathrm{MeV}$ for calorimetric energy deposits and than $50 \mathrm{MeV} / \mathrm{c}$ for charged particle transverse momenta. For the monojet search, at least one of the following trigger conditions was required to be satisfied: a total energy in excess of $6 \mathrm{GeV}$ in ECAL, or a track segment in the ITC matching an energy deposition of at least $1.3 \mathrm{GeV}$ in ECAL or a penetrating particle pattern in HCAL.

In the investigation of monojet final states, for which a clean missing energy identification is essential, the redundancy of energy measurements provided by the ALEPH detector is especially valuable: the energy in ECAL, a lead/proportional-wire-chamber sandwich, is measured independently in each of the 36 modules on the anode wire planes and on the cathode pads arranged in projective towers; the inactive regions at the boundaries between modules are backed by active regions in HCAL; the energy in HCAL, an iron/streamer-tube sandwich, is also measured independently by the tubes and by cathode pads grouped in projective towers. In each event, the energy flow is obtained by summing three components: i) charged particles reconstructed in the tracking system; ii) photons measured in ECAL; iii) neutral hadrons measured in both ECAL and HCAL. Double counting among calorimetric measurements and charged particle energies is avoided by the algorithm described in Ref. 4 , and the total energy resolution achieved is $\Delta E / E=60 \% / \sqrt{E}$ ( $E$ in $\mathrm{GeV})$. 
Using the energy and momenta of the charged and neutral particles delivered by the energy flow algorithm, the following criteria are applied to select monojet candidates.

Events with a minimum of two oppositely charged particle tracks are retained and, in order to ensure a good containment of the final state, it is required that no energy be detected within $12^{\circ}$ of the beam axis and that the total visible momentum point more than $25.8^{\circ}$ away from that axis. The small inefficiencies associated to this and to other energy vetos mentioned further down, due to occasional spurious or fake energy deposits in the calorimeters, have been monitored using events recorded at random beam crossings.

The monojet topology is enforced by the requirement that no energy should be detected in the hemisphere opposite to the direction of the total visible momentum. Since events resulting from photon-photon collisions indeed tend to exhibit such a topology because of spectator electrons remaining undetected in the beam pipe, a similar criterion is applied in the projection transverse to the beam axis: using only momentum components transverse to that axis, the "circularity" axis (the equivalent in two dimensions of the usual sphericity axis) is defined; the event is divided into two hemispheres by a plane perpendicular to that axis; and one of the two hemispheres thus defined is required to contain no energy. The few events from $\gamma \gamma$-interactions expected to remain at this level are eliminated by the requirement that the momentum component transverse to the beam axis should exceed $5 \% \sqrt{s}(\sqrt{s}$ is the centre-of-mass energy).

Monojets containing exactly three charged particle tracks are rejected. This introduces only a small inefficiency for real monojets, which should be electrically neutral, while removing the otherwise unavoidable background coming from $\tau$ pair events in which decay neutrinos take away essentially all of the energy of one of the $\tau$ s while the other one decays in the three charged prong topology.

Seven candidate events survive at this point, of which four are eliminated by the requirement that they should not consist of a single $\mathrm{e}^{+} \mathrm{e}^{-}$pair compatible with originating from a photon conversion. In particular, the pair invariant mass must exceed $200 \mathrm{MeV} / c^{2}$. This is in agreement with an expectation of 3.5 such events due to the process $\mathrm{e}^{+} \mathrm{e}^{-} \rightarrow \gamma \nu \bar{\nu}$ in which the photon converts in the detector material.

This selection finally leads to three monojet events:

- the $\mathrm{e}^{+} \mathrm{e}^{-}$pair reported last year;

- a hadronic system with two reconstructed charged particles, with a mass of $3.2 \mathrm{GeV} / c^{2}$ and a moderate $p_{T}$ of $6.6 \mathrm{GeV} / c$;

- a $5.3 \mathrm{GeV} / c^{2}$ mass hadronic system with a $p_{T}$ of $18.5 \mathrm{GeV} / c$. This event is shown in Fig. 1.

The masses of the invisible systems recoiling against the monojets are 61,80 , and $69 \mathrm{GeV} / c^{2}$, respectively. 
In fully simulated Monte Carlo samples of all the major standard processes $\left(\mathrm{e}^{+} \mathrm{e}^{-} \rightarrow \mathrm{ff}\right.$ and $\gamma \gamma \rightarrow \mathrm{ff}$, where $\overline{f f}$ is any quark or lepton pair), each corresponding to an integrated luminosity at least as large as for the data, no candidate events were selected by these criteria, and no events lay uncomfortably close to any particular cut. Because of the unpractically large Monte Carlo statistics which would be needed to reach a sensitivity level corresponding to, say, a few hundredths of events in the data, reasonable extrapolations and common sense arguments have to be used in order to conclude that these background sources indeed contribute to the selected sample at a level which is of no consequence for the analysis reported here. For instance:

- The number of events in which a lepton pair or a jet recoils against a purely neutral system is found to be $\sim 10^{3}$ in the data. This sample includes in particular the highly radiative ff $\gamma$ events. The probability for an electron, a muon or a charged hadron to leave no detectable signal in the electromagnetic and hadronic calorimeters is determined to be less than $10^{-5}$ for energies above $20 \mathrm{GeV}$, by inspection of the calorimetric signals associated to reconstructed charged particle tracks. The probability for a neutral hadron not to experience any inelastic interaction in the calorimeters is $\sim 10^{-3}$. Therefore, taking into account the composition of the neutral systems, the number of monojet events from this source where the neutral system would escape detection is expected to be smaller than 0.01 . In addition, the vast majority of these events would have small missing masses, in contrast to the events selected.

- Two monojets in the three charged track topology are observed in the data. This is in agreement with the expectation from the $\mathrm{e}^{+} \mathrm{e}^{-} \rightarrow \tau^{+} \tau^{-}$Monte Carlo, with three times more statistics than in the data. (The invisible $\tau$ decays are all due to $\tau \rightarrow \mathrm{e} \nu \bar{\nu}$, the only channel for which the final state can fall below the energy and momentum detection thresholds). Folding in the ratio of the known five-prong and three-prong topological $\tau$ branching ratios, $\sim 0.02$ events from this source are expected. But these events would not show up as $\mathrm{e}^{+} \mathrm{e}^{-}$pairs nor as monojets with masses in excess of $3 \mathrm{GeV} / c^{2}$.

- Low mass and acollinear $\mathrm{e}^{+} \mathrm{e}^{-}$and $\mu^{+} \mu^{-}$pairs, which are expected to arise from $\gamma \gamma$ interactions, can be used to determine the probability for a low polar angle energetic electron not to be detected in the electromagnetic or luminosity calorimeters. With a transverse momentum of the pair in excess of $5 \% \sqrt{s}$, the cut applied in the monojet search, this probability is found to be less than $10^{-3}$ (this estimate is statistically limited). This probability can then be folded with the number of data events which would be selected as monojets if the energy deposits at low polar angles were ignored. This leads to an expectation smaller than a few units in the data, falling below 0.1 events for $p_{T}>10 \mathrm{GeV} / c$, and well below 0.01 events for $p_{T}>15 \mathrm{GeV} / c$.

Therefore, the interpretation of the two highest $\mathrm{p}_{T}$ monojet candidates in terms of the standard $\mathrm{e}^{+} \mathrm{e}^{-} \rightarrow \overline{f f}$ and $\gamma \gamma \rightarrow$ ff processes is extremely difficult. The above arguments do not forbid that the lowest $p_{T}$ hadronic monojet be due to a $\gamma \gamma$ interaction, but it has been checked that the conclusions drawn below would be unaffected if this were actually the case. 
The simplest standard model interpretation for such monojet events is that they are due to the process $\mathrm{e}^{+} \mathrm{e}^{-} \rightarrow \gamma^{*} \nu \bar{\nu}$, with $\gamma^{*} \rightarrow \mathrm{ff}$, as depicted in Fig. 2a. The FERMISV four-fermion event generator[5], in which this specific process can be selected, has been used in order to check this hypothesis. In the case of the $q \bar{q} \nu \bar{\nu}$ final states, a weight has been applied to the generated events in order to take into account the experimental value of the ratio of the $\mathrm{e}^{+} \mathrm{e}^{-} \rightarrow$ hadrons cross-section at the $\mathrm{q} \overline{\mathrm{q}}$ mass to the prediction from the quark-parton model. After full simulation of the detector response and after the above described selection criteria have been applied, a total of 2.6 events are expected to be found in the data, which is in good agreement with the observation of three events.

However, as can be seen in Fig. 3, the mass and $p_{T}$ distributions of monojets from the process $\mathrm{e}^{+} \mathrm{e}^{-} \rightarrow \gamma^{*} \nu \bar{\nu}$ are peaked at low values. Low transverse momenta are indeed expected for photons from initial state radiation, and virtual photons tend to have low masses. In these respects, two of the three selected events are fairly atypical. To quantify the agreement or disagreement between expectation and observation, the procedure advocated in Ref. 6 has been applied:

- For each $\gamma^{*} \nu \bar{\nu}$ Monte Carlo event, with mass $m$ and transverse momentum $p_{T}$, the fraction $f$ of events in the Monte Carlo sample with mass larger than $m$ and transverse momentum larger than $p_{T}$ is determined; this provides the expected $f$ distribution.

- For each observed event, with mass $m_{i}$ and transverse momentum $p_{T i}$, the probability $y_{i}$ that the $\mathrm{e}^{+} \mathrm{e}^{-} \rightarrow \gamma^{*} \nu \bar{\nu}$ process lead to an at least as unlikely configuration of mass and transverse momentum is determined as the fraction of Monte Carlo events such that $f<f_{i}$, where $f_{i}$ is the fraction of Monte Carlo events with $m>m_{i}$ and $p_{T}>p_{T i}$.

- For $n$ observed events, the overall probability $\mathcal{P}$ of an at least as unlikely set of individual probability values $y_{i}$ is defined as the probability to observe an as low or lower value of the product of independent probabilities $Y=\prod_{i=1}^{n} y_{i}$; a simple calculation leads to the result $\mathcal{P}=Y \sum_{i=0}^{n-1}(-\log Y)^{i} / i$.

For the three events observed in the data, the probability $\mathcal{P}$ thus obtained is $1.0 \%$.

At such a level of probability, it is no longer sufficient to consider the process $\mathrm{e}^{+} \mathrm{e}^{-} \rightarrow$ $\gamma^{*} \nu \bar{\nu}$ as the only standard model source of monojet events. In the FERMISV generator, all diagrams involving photon or $\mathrm{Z}$ boson exchanges and leading to four-fermion final states can simultaneously be taken into account, including their interferences. These diagrams fall into four classes, shown in Fig. 2: the conversion diagrams (Fig. 2a), responsible for the process $\mathrm{e}^{+} \mathrm{e}^{-} \rightarrow \gamma^{*} \nu \bar{\nu}$ considered up to now; the annihilation diagrams (Fig. 2b); the bremsstrahlung (Fig. 2c) and multiperipheral (Fig. 2d) diagrams which contribute only to final states involving an $\mathrm{e}^{+} \mathrm{e}^{-}$pair. In the present discussion, the annihilation diagrams are particularly relevant: in spite of their small overall contribution, with 0.06 events expected, they tend to populate the high mass and high $p_{T}$ region, so that the probability $\mathcal{P}$ increases to $2.2 \%$. 
But there are still further diagrams leading to similar final states, not taken into account in FERMISV, and potentially contributing at a level similar to that of the annihilation processes. These diagrams, a sample of which is presented in Fig. 4, involve W exchanges.

The set shown in Fig. 4a, similar to the annihilation diagrams of Fig. 2b, has been considered in Ref. 7, together with $Z$ annihilation into a $W^{*} W^{(*)}$ pair (Fig. 4b). (The contribution to $\mathrm{W}$-pair production from $t$-channel neutrino exchange can be safely neglected at centre-of-mass energies close to the $\mathrm{Z}$ mass). The total number of monojet events expected from this source is 0.08 , concentrated at high mass and high $p_{T}$. When the ff' system in Figs. $4 \mathrm{a}$ and $4 \mathrm{~b}$ is a lepton-neutrino pair, the topology can be similar to the selected $\mathrm{e}^{+} \mathrm{e}^{-}$ monojet; when the $\mathrm{ff}^{\prime}$ 'system is hadronic instead, the mass recoiling to the $\mathrm{ff}^{\prime}$ ' $\mathrm{system}$ is small, thus rendering unlikely the interpretation of the selected hadronic monojets in terms of this process.

The diagram shown in Fig. 4c, similar to the bremsstrahlung process of Fig. 2c, can also lead to a monojet topology, especially since the spectator electron tends to remain undetected in the beam pipe. Together with the photon-W fusion diagram (Fig. 4d), this process is incorporated in the PYTHIA generator[8], following the calculation of Ref. 9. This leads to an expectation of 0.03 additional monojet events, populating the intermediate mass and $p_{T}$ region. The kinematic features of the highest $p_{T}$ hadronic monojet are similar to those expected from this source.

No other processes were found which could contribute in a similarly significant fashion to the monojet topology. Interferences within each of the three sets of diagrams shown i) in Fig. 2, ii) in Figs. 4a and 4b, and iii) in Figs. 4c and $4 \mathrm{~d}$ have been taken into account, but not among diagrams belonging to different sets. With this reservation, ${ }^{\dagger}$ the total number of monojet events expected is 2.75 , with mass and $p_{T}$ distributions as shown in Fig. 3. The probability $\mathcal{P}$ that the three events observed show up in as unlikely a configuration of masses and transverse momenta is $4.8 \%$.

In conclusion, three monojet events have been observed, all with masses larger than $3 \mathrm{GeV} / \mathrm{c}^{2}$. Two of these events have unexpectedly large transverse momenta: 18.5 and $20.3 \mathrm{GeV} / c$. The number of events observed is in good agreement with the expectation from the $\mathrm{e}^{+} \mathrm{e}^{-} \rightarrow \gamma^{*} \nu \bar{\nu}$ process, with $\gamma^{*} \rightarrow$ ff, but not their kinematic characteristics. When processes involving further $\mathrm{Z}$ or $\mathrm{W}$ exchanges are taken into account, the probability of observing an at least as unlikely configuration of masses and transverse momenta increases markedly but remains at the $5 \%$ level.

Acknowledgements: We wish to thank our colleagues from the accelerator divisions for the successful operation of LEP. We are indebted to the engineers and technicians in all our institutions for their contribution to the good performance of ALEPH. Those of us from non-member countries thank CERN for its hospitality.

$\dagger$ Upon completion of this work, we learnt of a recently released event generator, EXCALIBUR[10], geared toward $\mathrm{e}^{+} \mathrm{e}^{-}$collisions at LEP 200 and beyond, and which incorporates all electroweak four-fermion processes. 


\section{References}

1. CELLO Coll., H.J. Behrend et al., Phys. Lett. B 161 (1985) 182;

HRS Coll., C. Akerlof et al., Phys. Lett. B 156 (1985) 271;

JADE Coll., W. Bartel et al., Phys. Lett. B 155 (1985) 288;

MAC Coll., W. Ash et al., Phys. Rev. Lett. 54 (1985) 2477;

MARK II Coll., G.J. Feldman et al., Phys. Rev. Lett. 54 (1985) 2289;

ALEPH Coll., D. Decamp et al., Phys. Lett. B 236 (1990) 233;

DELPHI Coll., P. Abreu et al., Nucl. Phys. B 342 (1990) 1;

L3 Coll., B. Adeva et al., Phys. Lett. B 248 (1990) 203;

OPAL Coll., M.Z. Akrawy et al., Phys. Lett. B 236 (1990) 224.

2. ALEPH Coll., D. Buskulic et al., Phys. Lett. B 313 (1993) 312.

3. ALEPH Coll. D. Decamp et al., Nucl. Inst. and Methods A 294 (1990) 121.

4. ALEPH Coll., D. Decamp et al., Phys. Rep. 216 (1992) 253;

ALEPH Coll., D. Buskulic et al., "Performance of the ALEPH detector at LEP", to be submitted to Nucl. Inst. and Methods, in preparation.

5. J. Hilgart, R. Kleiss and F. Le Diberder, Comp. Phys. Comm. 75 (1993) 191.

6. F. Le Diberder, "Rarity and exoticness", MARK-II/SLC note 245 (1989).

7. V. Barger and T. Han, Phys. Lett. 241 (1990) 127.

8. H.-U. Bengtsson and T. Sjöstrand, Comp. Phys. Comm. 46 (1987) 43;

T. Sjöstrand, PYTHIA 5.6 Manual, CERN-TH.6488/92.

9. E. Gabrielli, Mod. Phys. Lett. A1 (1986) 465.

10. F.A. Berends, R. Pittau and R. Kleiss, "All electroweak four fermion processes in electron-positron collisions", INLO-PUB-1/94 and NIKHEF-H/94-08, April 22, 1994. 


\section{Figure Captions}

1. The highest mass and highest $p_{T}$ hadronic monojet selected.

2. Diagrams taken into account in the FERMISV generator:

a) conversion; b) annihilation; c) bremsstrahlung; d) multiperipheral. Unlabelled vector boson propagators correspond to $\gamma / \mathrm{Z}$ exchange.

3. Expected mass ( $\mathrm{a}$ and $\mathrm{b})$ and $p_{T}(\mathrm{c}$ and $\mathrm{d}$ ) distributions, absolutely normalized, calculated using all the diagrams shown in Figs. 2 and 4 (see text). The contribution from the process $\mathrm{e}^{+} \mathrm{e}^{-} \rightarrow \gamma^{*} \nu \bar{\nu}$, i.e. from the conversion diagrams of Fig. 2a, is shown shaded. The locations of the three selected events are indicated by arrows in a and $c$. The vertical scales are linear in a and $c$, logarithmic in $b$ and $d$; the horizontal scales have smaller ranges in a and $c$ compared to $b$ and $d$.

4. Diagrams involving $\mathrm{W}$ exchanges and contributing to the production of four-fermion final states. 


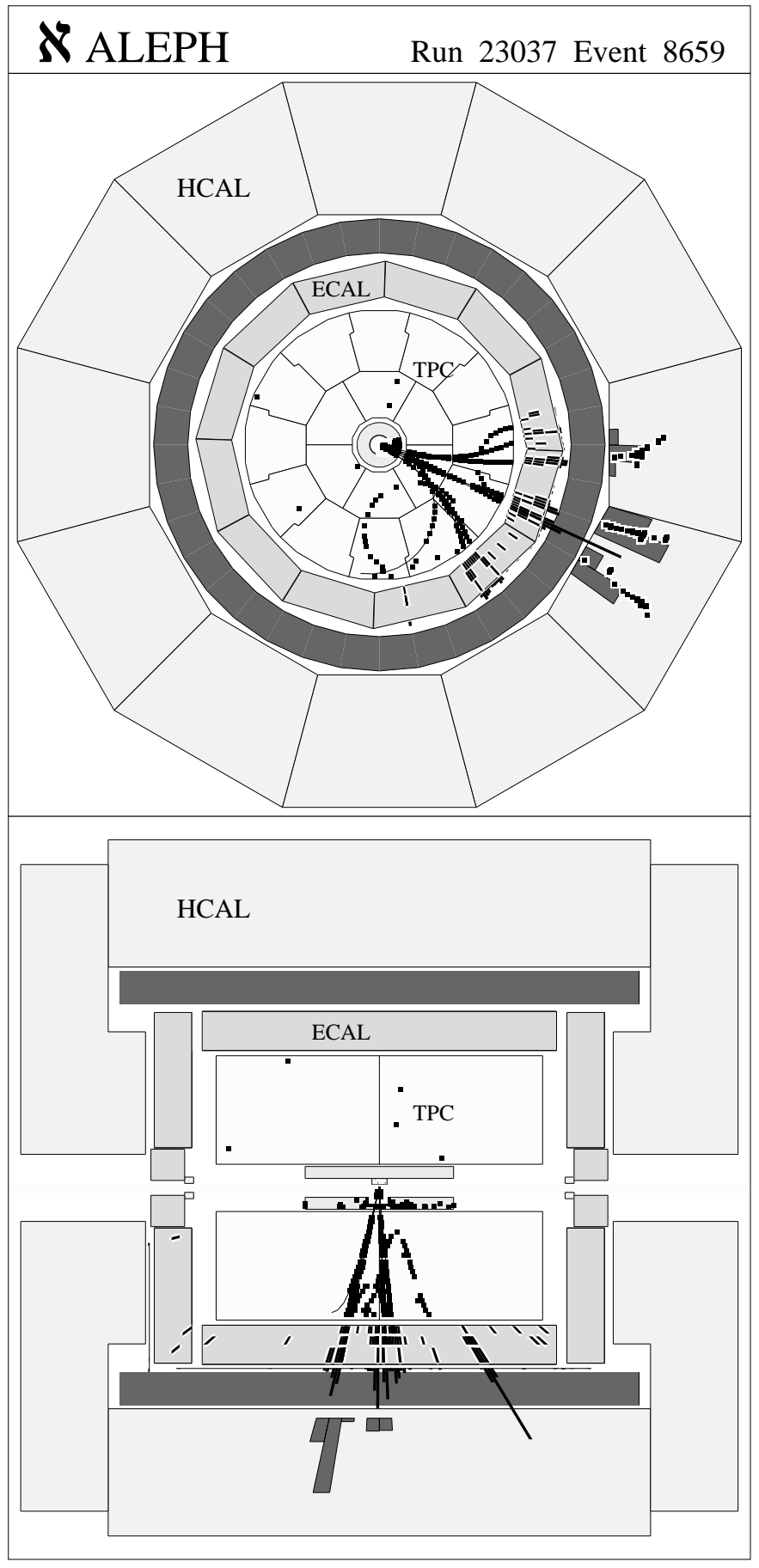

Figure 1 

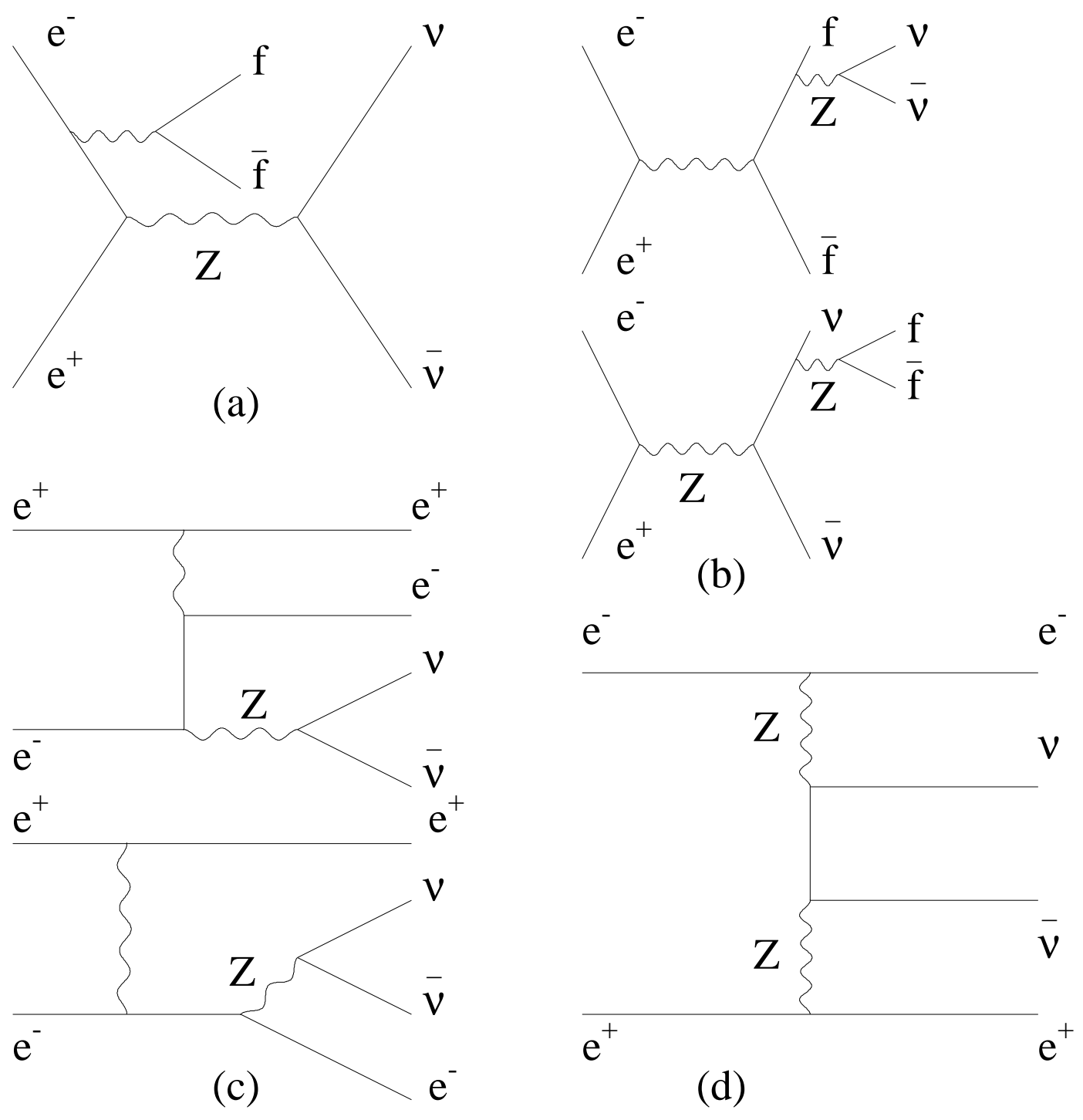

Figure 2 

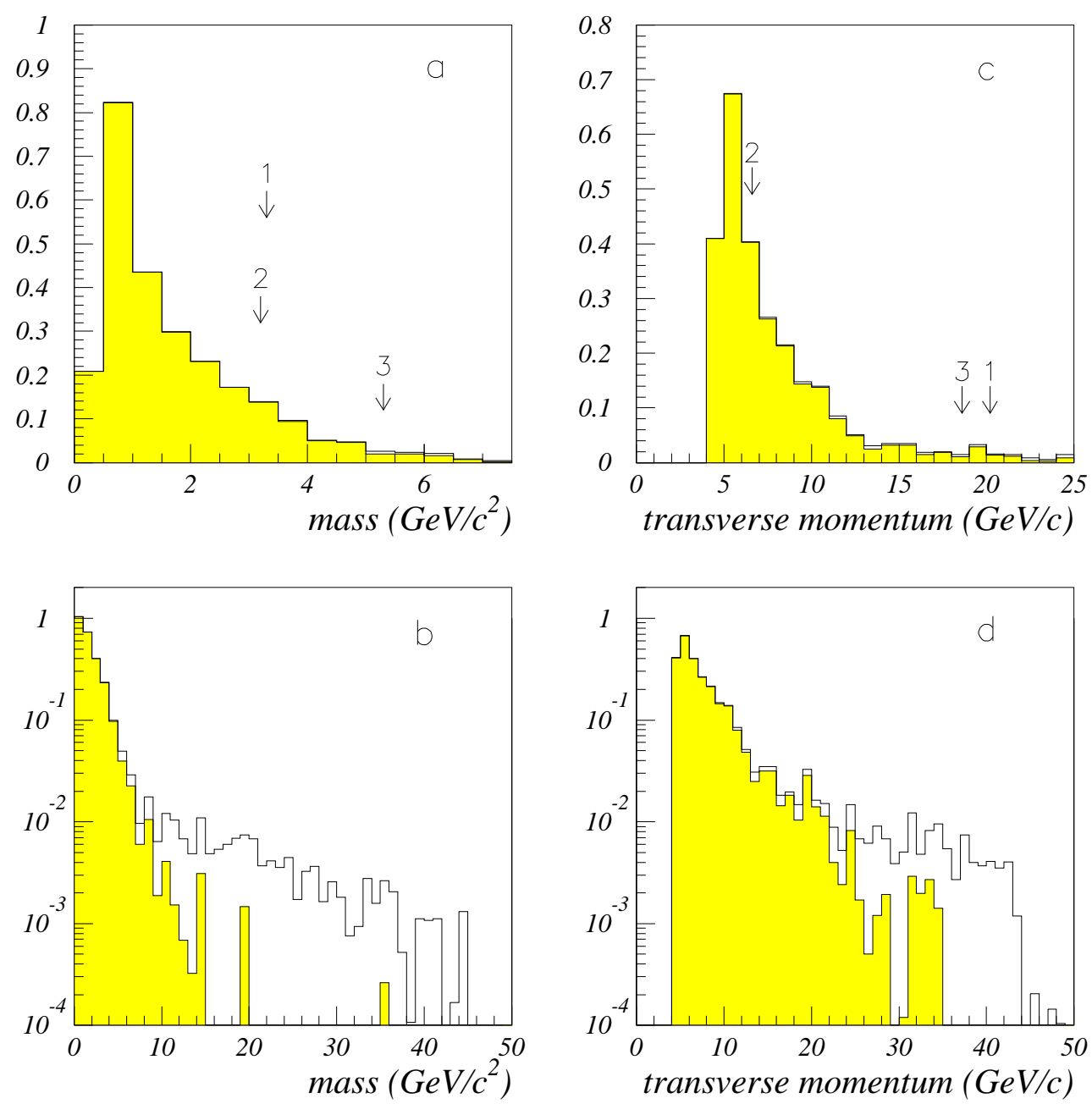

Figure 3 


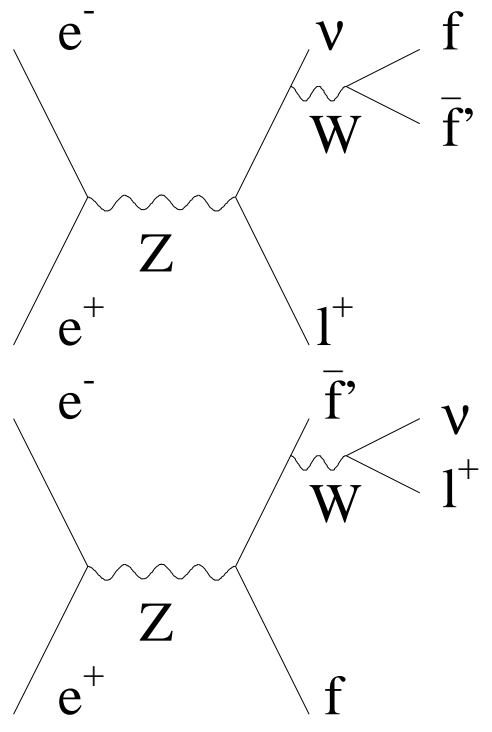

(a)

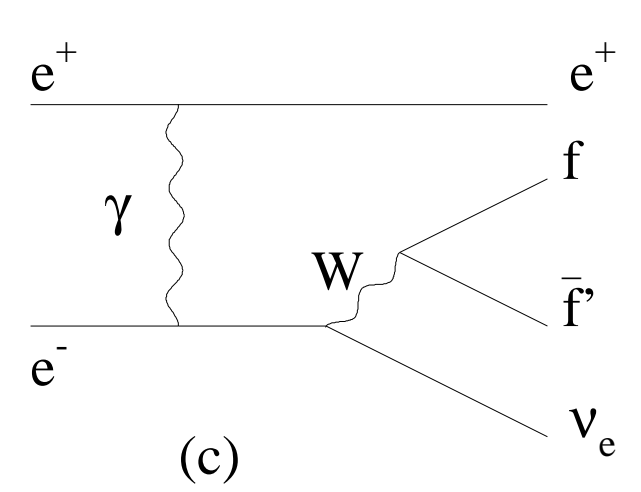

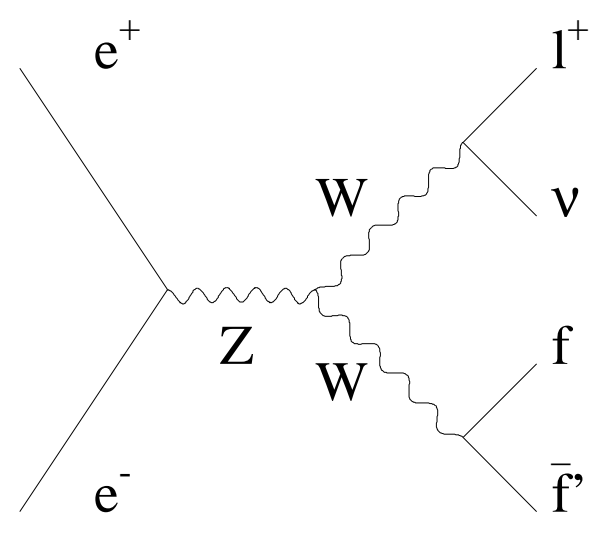

(b)

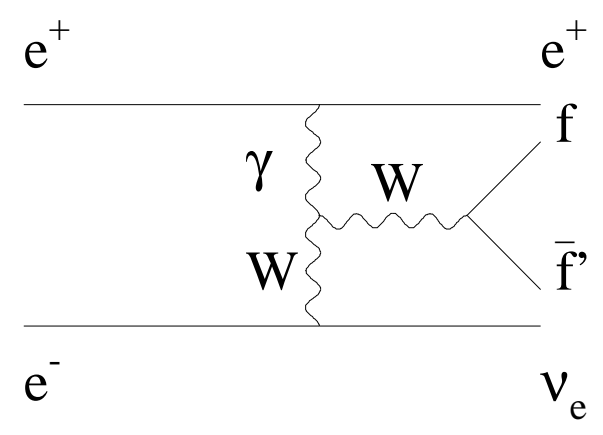

(d)

Figure 4 